\title{
MODELING AND ANALYSIS OF POLYPROPYLENE FIBER EXTRUSION PROCESS
}

\author{
Yousif A.A, Seedahmed A.I, Muhab.Hassanien S.S \\ Sudan University of Science and Technology, \\ Department of Polymer Engineering, Sudan
}

\begin{abstract}
Extrusion processes are widely used in plastic industries that aim to produce advanced solutions for increasingly sophisticated demands for plastics. Processes are not designed based on professional engineering design process, but on trial and error techniques. These two factors affect the extrusion processing parameters. In this study focus will be concentrated on the parameters incorporated in Polypropylene rope extrusion process such as: screw length, screw diameter, metering zone length and flight depth. A model equations were derived considering the swelling occurs for the extruded fibers and the tensile drawing of fiber. The authenticated design parameters was derived and a model useful to simulate any given process successfully was resulted in a form of computer software system.
\end{abstract}

Keywords - extrusion, polypropylene fiber, Swelling Ratio, Modeling and Analysis.

\section{INTRODUCTION}

For many years, polypropylene (PP) has been very successfully used for film blown, injection molded, and extrusion applications. [1] The interest in polypropylene(PP) is specifically due to the fact that the polymer is widely used as important engineering materials in the automotive, electrical appliances and packaging industries due to their excellent properties such as rigidity and stiffness, oil resistance and their thermal stability. [2]

Extruders are common devices in the plastic, metal, and food processing industries, and the utilization of extrusion processes is particularly widespread in product manufacturing that uses polymers as a raw material. Typical products made from extruded polymers includes, for example, pipes, hoses, insulated wires, cables, sheets, films, and tiles. [3-4]

Many theoretical, experimental, modeling and simulation studies have already described the polymer behavior in Extrusion process focusing on the polymer flow inside the barrel. [5]

Computer and its applications have entered various fields of applied sciences and engineering, for instance, Computer Aided Design (CAD) is being efficiently used for devices and equipment design, reducing the design calculations complexity in reliable manner. Regarding to polymers engineering, which deals with devices, equipment and polymer materials together, it uses in order to facilitate and accelerate the process of conducting engineering calculations. [6]

The design of polymer processing is currently supported by computer simulations based on the mathematical models of manufacturing processes. Modeling makes it possible to predict the course of these processes on the basis of process data (material, operation, and geometry). Modeling and simulation of polymer melt to produce fibers which are then used in ropes is a subject of this dissertation. The objective of this work to analyze the factors that affect the process in each step in the manufacturing sequence. [3]

Many research about screw design has been conducted. Experimental analysis for extrusion screw design to produce polypropylene fibers was investigated. The observation was experimentally conducted by using many screws with various geometries to obtain a highly transparent polypropylene melt resin sheet. The pressure distribution in the extruder, melt temperature profile across melt flow, the extruder throughput and the specific energy consumption were monitored. The work obtained screw geometry optimization was conducted using the analysis of melting performance by the cooling experiment and the pressure pattern. As a result, the screw geometry to satisfy a low external haze and extrusion stability under higher throughput conditions was designed. [7]

[Kaiserslautern (2001) simulated the influence of process parameters to the melt spinning process a fiber model is used and coupled with CFD calculations of the quench air flow. In the fiber model energy, momentum and mass balance are solved for the polymer mass flow. [8] 


\section{International Journal of Engineering Applied Sciences and Technology, 2021 \\ Vol. 5, Issue 12, ISSN No. 2455-2143, Pages 317-321 \\ Published Online April 2021 in IJEAST (http://www.ijeast.com)}

[Doufa (2001) studied the temperature field subjected to an exponential stretching rate. The numerical analysis of melt spinning was also extended from isothermal Newtonian flow to viscoelastic fluid, non-isothermal Newtonian flow, non-isothermal viscoelastic flow and recently flow with crystallization [9]

A. Lewandowski (2019) applied ANSYS Polyflow software to model the melt flow in the extruder and analyzed the flow of polymers with slip effects both in the screw (on the screw and barrel surfaces) and in the die. Screw pumping characteristics and die characteristics are calculated and modeled for various power law indices, and various slipping parameters. The effect of slipping on extruder operation is discussed. [10] Tingrui (2017) used a statistical, design of experiments (DoE) approach. For a conventional screw design, barrel temperature, screw speed and two vastly different melt viscosity PP mixtures were selected as the independent factors, whilst melt pressure, mass output, screw torque and temperature rise at the die due to shear heating, were the dependent responses [11]

ZHOUKE (2014) used POLYFLOW simulation plat form to model and analyzed the single screw extrusion process of propellant through the application of Finite Element Analysis on extrusion of plastic. The distributions and changes of viscosity in extrusion process [12]

A. Farhoumand (2016) were applied a finite element method to investigate the effect of process parameters of plastic deformation behavior in Forward-BackwardRadial Extrusion (FBRE) process. The result of an ax symmetric model shows that the friction between die components and the sample has a substantial effect on the material flow behavior. [13]

W.E. Abdel-Ghany (2015) investigated the characteristics of single screw extruders and evaluate the performance of various types of screw under different working conditions using finite element analysis. [14] In this paper focus on modeling and analysis of polypropylene fibers extrusion process and model work is done using $\mathrm{C \#}$ language to model polypropylene fibers process design in multi stage from screw to draw unit.

\section{MODELING METHODS:}

Most of the lines that produce fibers for rope manufacturing has the capacity of $65 \mathrm{~kg} / \mathrm{h}$. This was used as the basis of the design to analyze the flow of the PP melt in order to specify the design features of the extruder, the extruder diameter and its length. Different design equations of the extruder were used to specify the three zones feeding, melting and metering zone. For each zone flight height was determined in addition to the required heating at melting zone and the length of each zone. Different design equations of the spinneret were used to analyze the swelling occurs in the extruded fibers and cooling process and then analyzed the following processes of heating and tensile drawing of fiber to obtain the required diameter for the use of ropes. C Sharp language was used to design a model to solve the parameters for any given process.

\section{A. extruder Analysis :}

extruder analysis depended on flow mechanism, drag flow and pressure flow for the determination of the screw length screw diameter, length of the three zones and the number of flight of these zones suitable for manufacturing polypropylene fibers process. [16]

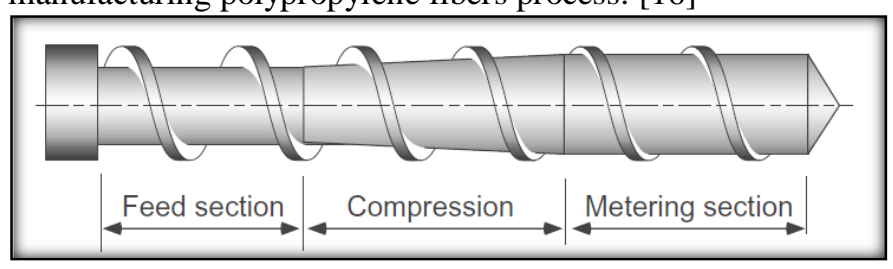

Fig.1. Screw Zones [15]

The polypropylene data as shown in table (1) are used to analyze and design the single screw extruder to determine the compression zone length suitable for polypropylene extrusion process [15-16]

Table 1: Specifications of Polypropylene

\begin{tabular}{|l|c|c|}
\hline \multicolumn{1}{|c|}{ Trade Name } & $\begin{array}{c}\text { The } \\
\text { Units }\end{array}$ & $\begin{array}{c}\text { The } \\
\text { Value }\end{array}$ \\
\hline Melting temperature at compression zone & $\mathbf{k g} / \mathbf{m 3}$ & $\mathbf{9 1 0}$ \\
\hline Constant temperature & $\mathbf{1 9 0}$ \\
\hline Heat diffusion & ${ }^{\circ} \mathbf{C}$ & $\mathbf{2 1 0}$ \\
\hline Medium temperature & $\mathbf{J} / \mathbf{k g}$ & $\mathbf{2 3 . 4 \times 1 0}$ \\
\hline temperature of fiber at leaving cooling bath & ${ }^{\circ} \mathbf{C}$ & $\mathbf{3 0}$ \\
\hline Thermal conductivity & $\mathbf{C} / \mathbf{m} \cdot \mathbf{k}$ & $\mathbf{0 . 1 7 2}$ \\
\hline Melting occurs at the adjacent film & $\mathbf{m m}-$ & $\mathbf{1}$ \\
\hline Mass Flow Rat & $\mathbf{K g} / \mathbf{h}$ & $\mathbf{6 5}$ \\
\hline Poisson's ratio & - & $\mathbf{0 . 4}$ \\
\hline Elastic Modula's & $\mathbf{N} / \mathbf{m}^{2}$ & $\mathbf{1 . 5 x 1 0}$ \\
\hline Glass transition temperature & ${ }^{\circ} \mathbf{C}$ & $\mathbf{- 1 0}$ \\
\hline Medium cooling temperature & ${ }^{\circ} \mathbf{C}$ & $\mathbf{3 0}$ \\
\hline Medium heating temperature & ${ }^{\circ} \mathbf{C}$ & $\mathbf{8 0}$ \\
\hline final temperature of the solid fiber & ${ }^{\circ} \mathbf{C}$ & $\mathbf{7 0}$ \\
\hline Initial temperature at heating bath & ${ }^{\circ} \mathbf{C}$ & $\mathbf{4 0}$ \\
\hline Melt draw ratio & - & $\mathbf{4}$ \\
\hline Thermal diffusely & & $\mathbf{1 0}{ }^{-7}$ \\
\hline
\end{tabular}




\section{B. Analysis of Melt Spinning:}

The objective from Analysis of Melt Spinning to analyze the factors that affect the process in each step in the manufacturing sequence and to determine its appropriate parameters for the process such as : Swelling Ratio ,Draw Ratio ,Force required to draw the melt , Force Required to draw the Soiled fiber, Drawing velocity of the fiber, Cooling bath length, heating bathe length and final fiber required dimensions. [15]

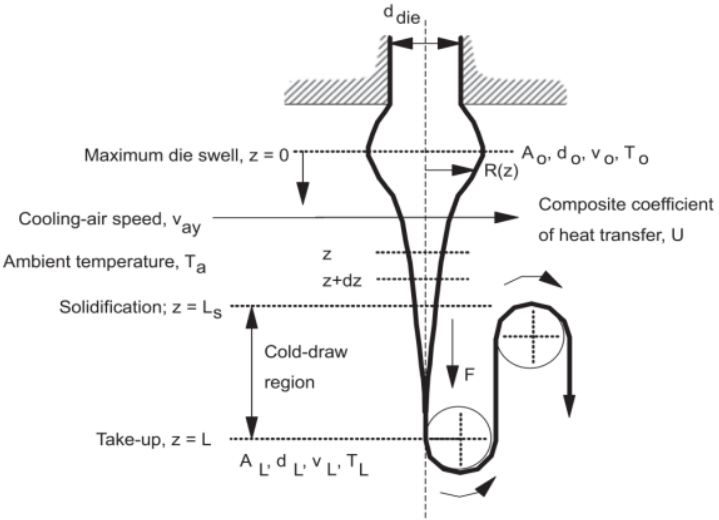

Fig.2 Analysis of melt spinning [15]

\section{Modeling Tools and Software}

In This study a computer program was used, namely $\mathrm{C}$ Sharp (C\#), to develop a software system capable of performing complex design calculations of the single screw extruder. beside analysis of the extrusion and spinning. All equations used to design the extruder and spinneret were used in C Sharp and a system model was designed in a form of application.as shown in the following figures.

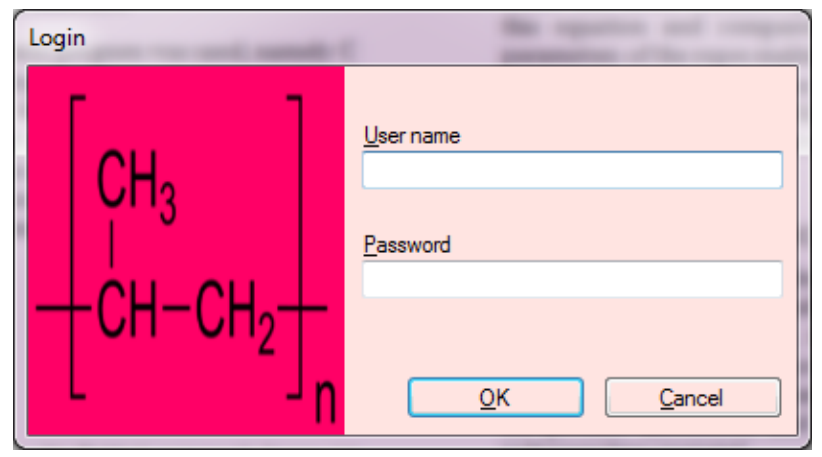

Fig. 3 login Screen

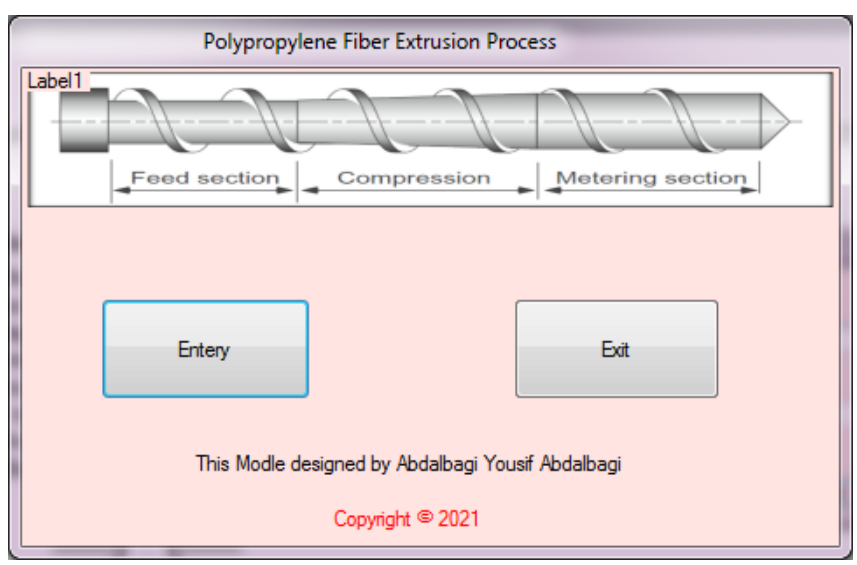

Fig.4 Main Screen

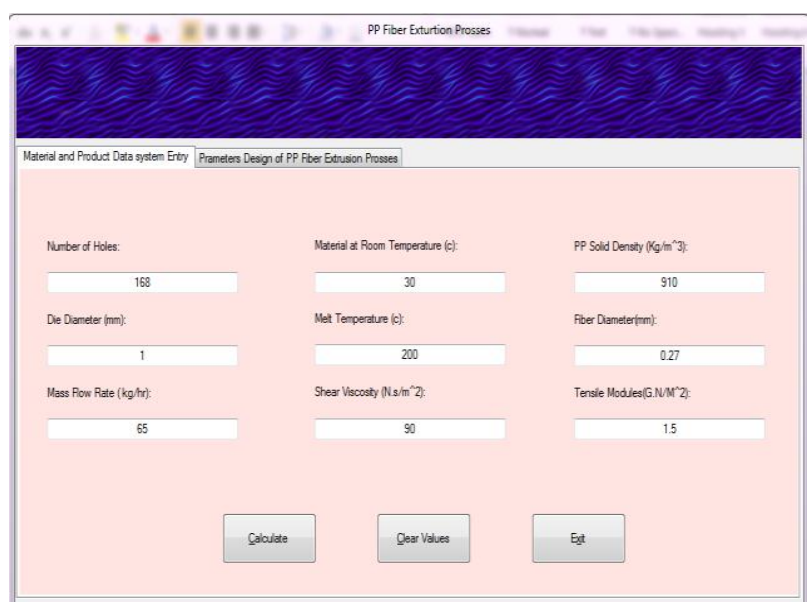

Fig.5 Inputs Screen

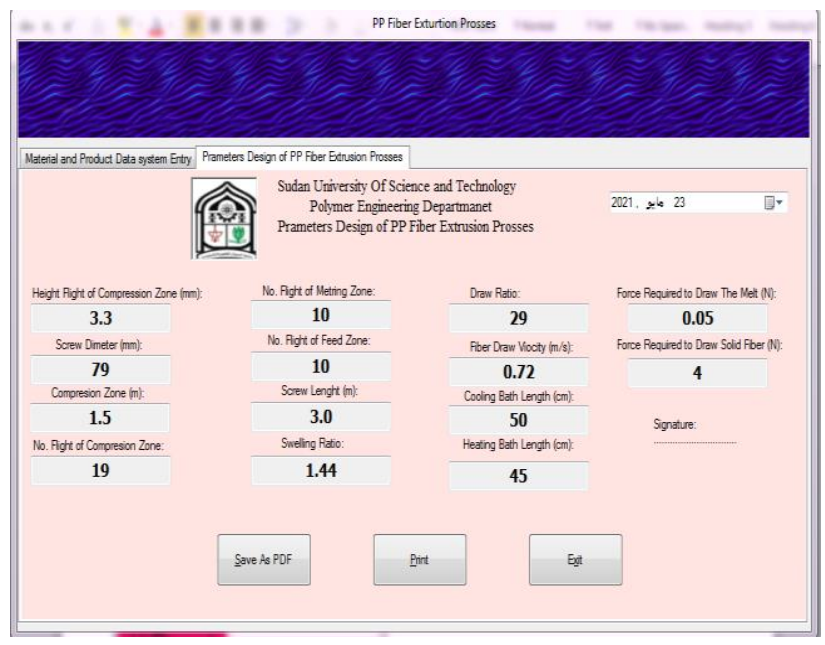

Fig.6 Outputs Screen 


\section{International Journal of Engineering Applied Sciences and Technology, 2021 \\ Vol. 5, Issue 12, ISSN No. 2455-2143, Pages 317-321 \\ Published Online April 2021 in IJEAST (http://www.ijeast.com)}

\section{RESULT AND DISCUTION}

Extruder and spinneret design most important in polypropylene fiber process for the determination of appropriate parameters for the process. The extruder analysis was depended on flow mechanism, consider the flow consider circular section most the flow produced from extruder is drag flow and pressure flow. Some considerations inherent in the melting and flow of the molten process were taken into account, such as the amount of heat required from the heaters and the resultant of the shear, the area to be heated, the heat transfer coefficient and the use of the data on the physical, thermal, and rheological properties of polypropylene to determine screw diameter, screw length, feed zone length, metering zone length, compression zone length, high flight of compression zone length, number flight of compression zone, number flight of feed zone and number flight of metering zone. For the determination of the final dimension of the fibers, some considerations such as Swelling ratio, heat transfer process at cooling and heating bath and tensile force. The fibers extrusion process was analyzed using this equation A application was designed that gave real results, the application allows documenting the result as a pdf or printing it and compared with the real value of parameters of the ropes making manufacturing that was manufactured by trial, error and experience, and the same result was obtained. Mechanism of the modeling software included all these design equations.

\section{CONCLUSION}

In this study, a model was designed to assist the designer of polypropylene fiber manufacturing machines used in the manufacture of ropes, as it helps in solving the problems facing maintenance and operator's engineers by determining the appropriate parameters according to the product dimensions and the specifications of the polypropylene material.

\section{REFERENCE}

[1] Shalby M. S. and Seedahmed A. I. (2014), Evaluation and Improvement of Mechanical, Thermal and Rheological Properties of Polypropylene (PP) using Linear Low Density Polyethylene (LLDPE), International Journal of Engineering Science and Innovative Technology (IJESIT), 3(3), 135- 141.

[2] Hassanien M S, Seedahmed A.I., (2015), Mechanical and Rheological Properties of Polypropylene(PP)/Linear Low Density Polyethylene(LLDPE) Blend Filled with Talc and Calcium Carbonate Compositions, International Journal of Engineering Sciences and Research Technology, 4(1), 383 - 387

[3] Yousif A.A. (2021). Determination And Optimization Of Compression Zone Length For Polypropylene Fiber Process, International Journal of Engineering Applied Sciences and Technology, Pages 27-29

[4] Crowley MM, Zhang F, Repka MA, Thumma S, Upadhye SB, Kumar Battu S, McGinity JW and Martin C .(2007). Pharmaceutical applications of hot-melt extrusion: part I. Drug development and industrial pharmacy 33:909926.

[5] Sauceau M, Fages J, Common A, Nikitine C and Rodier E .(2011). New challenges in polymer foaming: A review of extrusion processes assisted by supercritical carbon dioxide. Progress in Polymer Science 36:749766.

[6] Hirz M, Dietrich W, Gfrerrer A and Lang J .(2013). Integrated computer-aided design in automotive development. Springer-Verlag, Berlin-Heidelberg, DOI 10:978-3.

[7] Funaki A, Takubo $\mathrm{T}$ and Kanai $\mathrm{T}$ .(2010).Experimental analysis for extrusion screw geometry to produce highly transparent polypropylene sheets. Polymer Engineering \& Science 50:420-427

[8] Kaiserslautern, im Juni .(2001). "Melt spinning, fiber model, Lattice Boltzmann, CFD" Journal of Rheology. 45(2): p. 403-420.

[9] Doufas, A.K. and McHugh, A.J.(2001). Twodimensional simulation of melt spinning with a micro structural model for flow-induced crystallization. Journal of Rheology,. 45(4): p. 855-879.

[10] Lewandowski A and Wilczyński K (2019) Global Modeling of Single Screw Extrusion with Slip Effects. International Polymer Processing 34:81-90.

[11] Fu T, Haworth B and Mascia L. (2017). Analysis of process parameters related to the single-screw extrusion of recycled polypropylene blends by using design of experiments. Journal of Plastic Film \& Sheeting 33:168-190.

[12] Ke Z, Zhongqi H, Shupan Y and Wanghua C. (2014). Numerical simulation for exploring the effect of viscosity on single-screw extrusion process of propellant. Procedia Engineering 84:933-939. 
[13] Farhoumand A and Ebrahimi R. (2016).Experimental investigation and numerical simulation of plastic flow behavior during forward-backward-radial extrusion process. Progress in Natural Science: Materials International 26:650-656.

[14] Abdel-Ghany W, Ebeid S and Fikry I .(2015).Mechanical Design Aspects of Single Screw Extruders using Finite Element Analysis. International Journal of Engineering and Technical Research (IJETR) 3:245 - 4698.

[15] [Baird DG and Collias DI .(2014). Polymer processing: principles and design. John Wiley \& Sons.

[16] Crawford RJ (1998) CHAPTER 5 - Analysis of polymer melt flow. In: Crawford RJ (ed)^(eds) Plastics Engineering (Third Edition), Butterworth-Heinemann, Oxford pp. 343-412 